\title{
A Point-of-Care Device for Sensitive Protein Quantification
}

\begin{abstract}
In this paper we present the design of a new pointof-care $(\mathrm{PoC})$ device for protein quantification. The proposed design is based on a novel aptamer-mediated methodology and real time polymerase chain reaction (RT-PCR), a robust and ultrasensitive method for DNA amplification, which we employ for very sensitive quantification of proteins. In addition, we have also developed an algorithm for the processing of raw fluorescence data from the portable RT-PCR device. The algorithm leads to better linearity than a proprietary software from a commercially available RT-PCR machine. The modular nature of the system allows for easy assembly and adjustedment towards a variety of biomarkers for applications in disease diagnosis and personalised medicine.
\end{abstract}

Index Terms-System design, point-of-care, RT-PCR, algorithm, noise minimisation

\section{INTRODUCTION}

Point-of-care $(\mathrm{PoC})$ tests refer to any diagnostic test done near the patient, without the need for laboratory facilities [1]. Most PoC devices for protein detection are based on the enzyme-linked immunosorbent assay (ELISA), which requires expensive equipment for detection and the use of a standard curve for quantification, with limits of detection restricted by the available equipment. An alternative approach could be to use aptamers. Aptamers are short single-strand sequences of DNA/RNA, and they offer many advantages compared to antibodies, including long shelf life and simple workflow [2], which make them particularly suitable for PoC devices. Moreover, being DNA sequences, they can be quantified through real time polymerase chain reaction (RT-PCR) [3], a robust and sensitive DNA quantification method which can theoretically detect up to a few sequences. Previous studies [4]-[7] have described portable and inexpensive RT-PCR machines, but did not include automated sample preparation. Toumazou et al. [8] proposed a device for automated sample preparation to be used with a portable RT-PCR machine, but the device only applies to DNA extraction and therefore, cannot directly be used for protein quantification.

The work presented in this paper is part of our development of an ultra-sensitive protein quantification system. Here we propose a device which is portable, while addressing the issues and challenges of implementation. The molecular methodology is described in [9], whereas here we present the design and technical aspects of our PoC device. The basis of our approach is the amplification of a chemical signal through the use of RT-PCR, which allows one to reliably detect very small protein concentrations. The device description is based on the quantification of the gut hormone leptin, an important protein involved in the regulation of energy balance which is associated to obesity and type 2 diabetes [10].

In principle, this technique can be used for very sensitive and accurate PoC antigen testing, as well as antibody testing for a variety of conditions, provided a suitable aptamer is available and target-specific sequences are designed. Such a device which would permit detection of very low levels of antibodies, is very much sought after in the current COVID19 pandemic.

\section{BACKGROUND}

\section{A. Aptamer-Adapter Complex methodology}

The aptamer-based method for ultrasensitive protein quantification is described in detail in [9]. A brief description is presented in Fig. 1.

\section{B. Aptamer-based PoC devices}

Work by Dhiman et al. [11] extensively described the applications of aptamer-based PoC devices, in which aptamers are usually employed as a direct substitute of antibodies. While most of the available $\mathrm{PoC}$ tests are inexpensive and can be operated directly by the patient, they rely on visual quantification which limits the sensitivity and resolution of the tests. However, when aptamers are considered as DNA sequences and PCR is used as the quantification method, sensitivity and resolution can be greatly improved.

\section{Techniques for analysis of RT-PCR data}

PCR is a technique that works by doubling target DNA sequences at each cycle until PCR resources (polymerase and primers) are depleted and a plateau is reached. In RT-PCR, fluorescence measurements are taken at each cycle, and a sigmoidal curve is generated (Fig. 1(E)). The point at which the fluorescence starts increasing significantly is called the 'threshold cycle' $\left(C_{t}\right)$-which is inversely proportional to the DNA concentration-and is used to quantify samples against a standard curve. This quantity is critical for quantifying the original amount of the targeted substance; therefore, its precise measurement is crucial.

There are numerous methods to obtain the $C_{t}$ value, with the simplest calculating the $C_{t}$ value the cycle at which the fluorescence signal crosses a constant threshold. Since this 


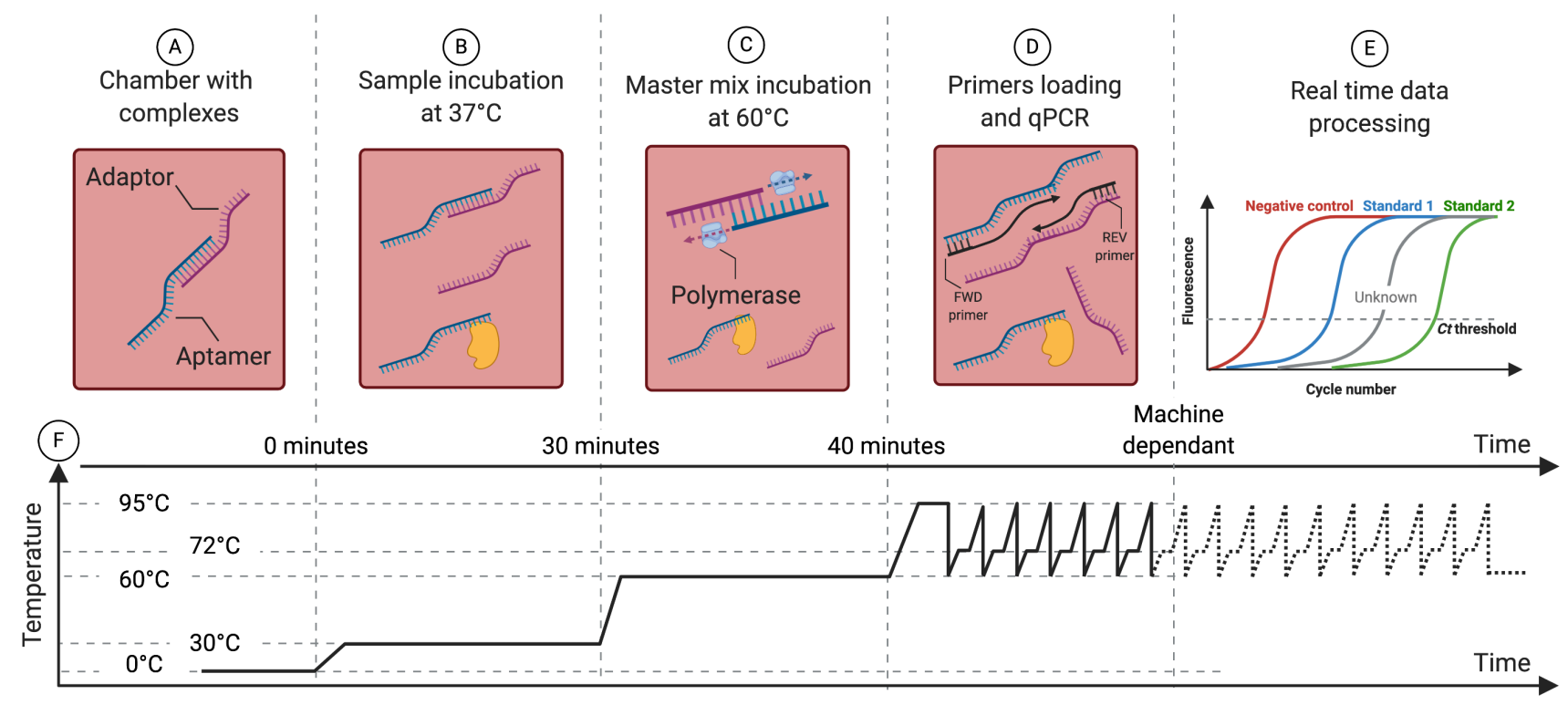

Fig. 1: (A-D) Sensing methodology: chambers are pre-loaded with complexes (A); sample containing target protein is loaded and displaces the complexes proportionally to its concentration (B); the master mix for complex elongation and PCR is loaded (C); the primers are loaded and PCR starts (D). (E) RT-PCR sigmoid surves. (F) Temperature profile of the machine for sample preparation and RT-PCR.

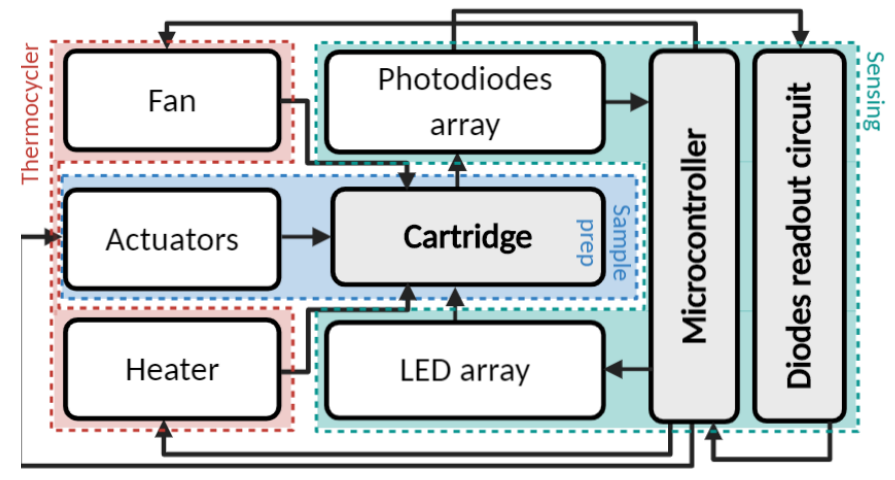

Fig. 2: Functional block diagram of the proposed system.

method is sensitive to scale, it requires data scaling, usually implemented through fitting to a logistic function [12]. Other methods define the $C_{t}$ as the cycle at which the $1^{\text {st }}$ or $2^{\text {nd }}$ derivatives reach the maximum. More complicated algorithms exist which achieve more precise estimates of $C_{t}$ [13], [14] but might be unsuitable for computation within a simple PoC device.

\section{PoC SYSTEM DESIGN}

The system implements the automated aptamer-based quantification method described above and in [9]. As shown in Fig. 2 , the system is composed of a sample preparation module (actuators and cartridge, blue shadow), a thermocycler (fan, heater and microcontroller, red shadow) to run the PCR, and a sensing and data processing module (photodiodes array, LED array, readout ciruit and processor, cyan shadow).
Below, we describe the design methodology for the cartridge and the data processing of the RT-PCR data.

\section{A. Cartridge}

The cartridge implements the aptamer-based method and in our case, it is using a leptin-specific aptamer, a complementary adaptor and primers specific to the complexes. Given the simplicity of this method that does not require discarding solutions, a simple microfluidic cartridge operated by linear actuators and air pumps can be implemented.

Fig. 3 shows the working principle of the cartridgde: upon sample loading (in the chamber 'Sample' in Fig. 3), the complexes (Fig. 3.1a) are displaced by leptin which attaches to the aptamers (Fig. 3.1b). Then, the polymerase extends the undisplaced complexes to form fully double stranded sequences (Fig. 3.2). Finally during PCR the primers attach specifically to the double stranded sequences without amplifying free adaptors or aptamers (Fig. 3.3).

The sample, the standards and the control are loaded in the chambers-pre-loaded with the hybridized aptamer-adaptor complexes ('Sample' and control chambers, Fig. 3)-and incubated at $37^{\circ} \mathrm{C}$ for 30 minutes (Fig. 1B). The master mix containing the polymerase and other PCR components is loaded and the solutions are incubated at $60^{\circ} \mathrm{C}$ for 30 minutes (Fig. 1C). Right before the PCR starts, the primers are loaded (Fig. 1D). The PCR happens in the same chambers array used for sample preparation.

The two blister pouches containing the master mix and the primer mix (Fig. 3) are operated by linear actuators, and air pumps allow the solutions to flow. The main challenge concerning the cartridge implementation is the design of suitable mixing mechanisms to achieve a uniform distribution of the 


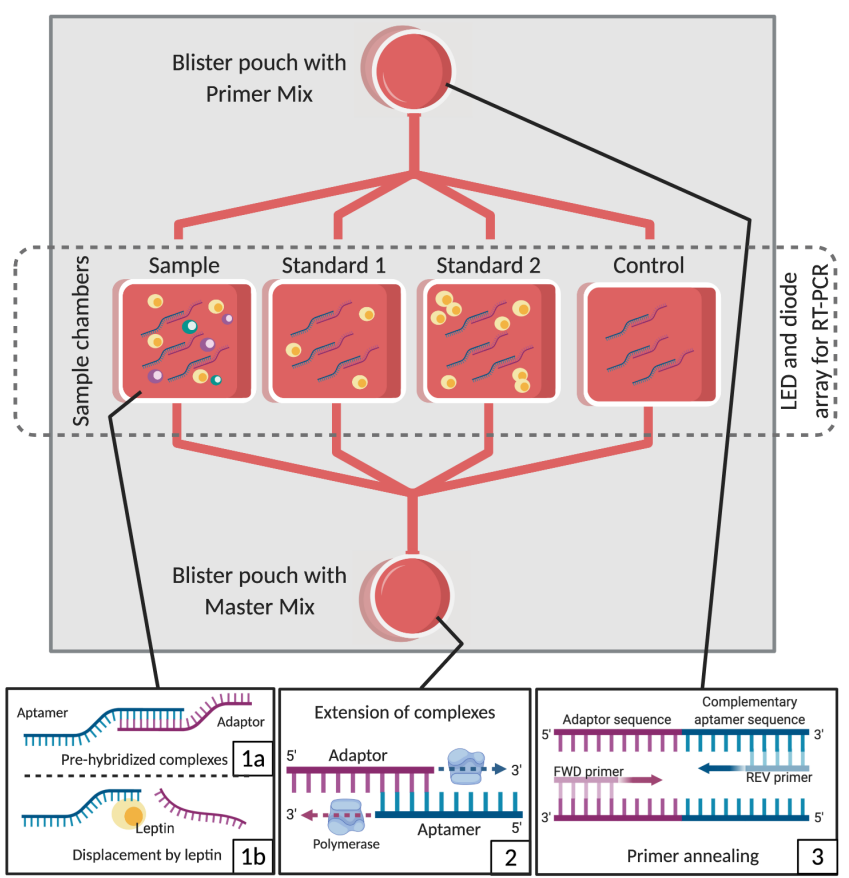

Fig. 3: Microfluidic cartridge and underlying biochemical process.

molecules in the chambers, which ensures proper interaction of the molecules. The shelf life of the master mix is a further challenge; to avoid storage in freezers, the components should be lyophilized and resuspended when running the samples. This would require including extra pouches (and corresponding actuators and pumps) containing the resuspension solution.

\section{B. $q P C R$ module}

The RT-PCR module is based on the design a PCR unit used for COVID-19 detection [15]. The module described in this paper is made of heating and cooling elements acting as a thermocycler for the cartridge, which is placed on an aluminum heat block. Contrary to usual RT-PCR machines, the present module includes a temperature profile for sample preparation (incubation with sample and with master mix), see Fig. 1(C), followed by pre-incubation at $95^{\circ} \mathrm{C}$ for 600 s and a three-step PCR amplification: DNA denaturation $95^{\circ} \mathrm{C}$ for $10 \mathrm{~s}$, primer annealing at $60^{\circ} \mathrm{C}$ for $10 \mathrm{~s}$ and DNA elongation at $72^{\circ} \mathrm{C}$ for $10 \mathrm{~s}$. At each annealing step, the LEDs shine blue light of wavelength $\lambda \approx 497 \mathrm{~nm}$ absorbed by the SYBR green dye, which emits fluorescence in proportion to the target DNA concentration in the chambers. The green light emitted by the dye $(\lambda \approx 520 \mathrm{~nm})$ is captured by the photodiodes and readout circuit.

The design of the RT-PCR machine affects the quantification performance greatly: thermal cycling speed impacts DNA amplification efficiency, and noise in the fluorescence detection affects the precision of the measurements [16].

\section{Data processing}

The fluorescence data is likely to contain the noise introduced during the biochemical process (due to mixing

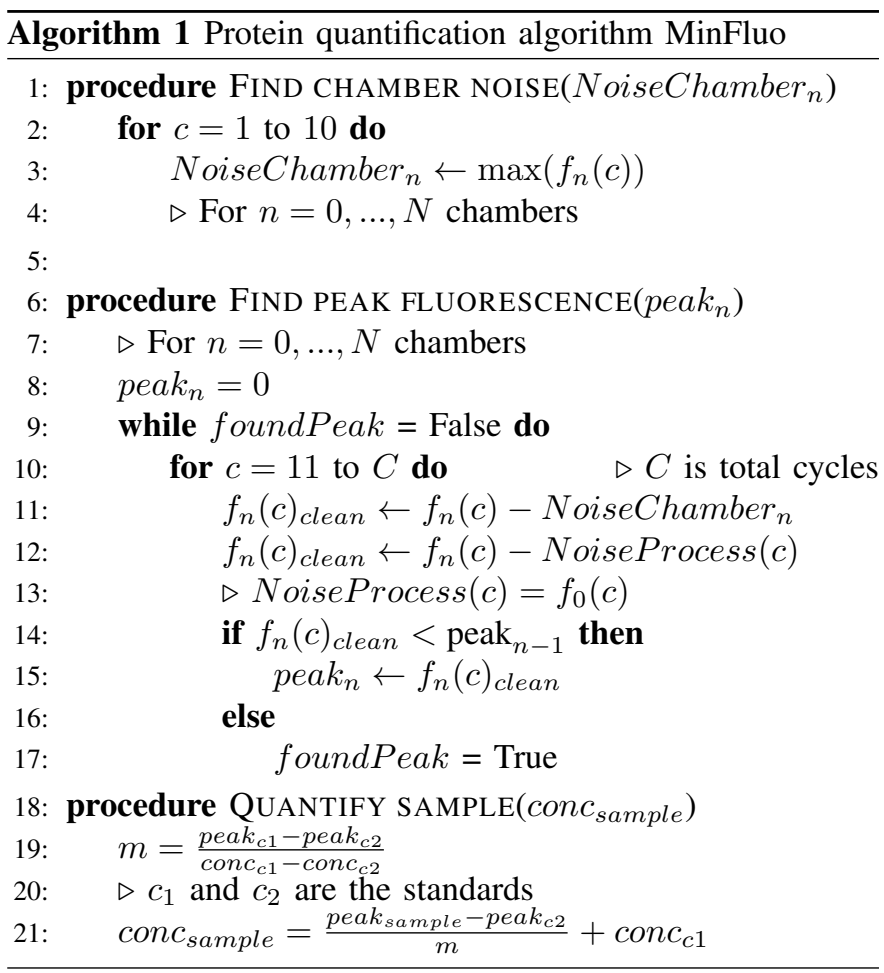

imperfections), during the PCR (due to device capabilities and reaction efficiency), and during the fluorescence reading (due to noise introduced by variations in photodiodes and LED light intensity). Given the absence of washing steps, the free sequences and proteins will remain in the chambers and are likely to inhibit PCR. To minimise the effect of noise introduced by the biochemical process (NoiseProcess), a control is included and subtracted from the fluorescence of the standards and samples.

The processing is done in real time while the PCR is running-the processing algorithm is shown above. First, the chamber noise NoiseChamber ${ }_{n}$ represents the noise of each reaction and is determined as the maximum fluorescence in the first 10 cycles. Then from cycle 11 at each cycle the chamber noise is removed from all signals including the control, and the process noise NoiseProcess is removed from the standards and unknowns. Given the convex nature of the cleaned fluorescence signal (see Fig. 4), the process stops once the global minimum is found for each fluorescence signal, thus reducing the RT-PCR running time.

Noise in the LED and photodiode variation can be minimised by splitting the final solutions with master mix and primer mix in each chamber into replicated and considering the average of the fluorescence across replicates.

\section{EXPERIMENTAL RESULTS}

Fluorescence data was collected with a commercially available qPCR machine. Fig. 4 shows the fluorescence signals after chamber and process noise removal, with the characteristic peak. A standard curve was build by fitting a linear model to the $\log$ (concentration) and the fluorescence at the minimum 


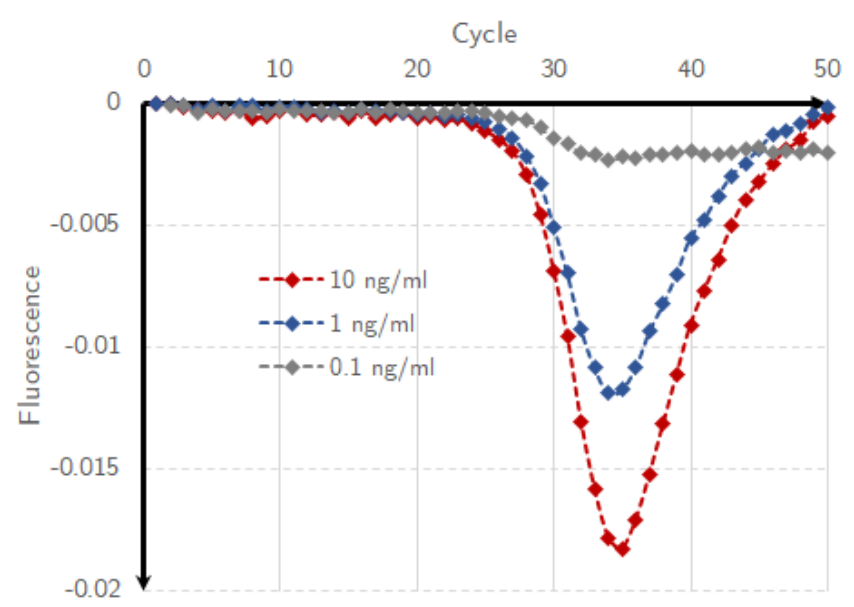

Fig. 4: Fluorescence curves after chamber noise and process noise (control) removal.

TABLE I: Comparison of $R^{2}$ achieved by MinFluo (the method presented in this paper) and by the processing done by a commercial software on three different experiments.

\begin{tabular}{cccc}
\hline & Exp. 1 & Exp. 2 & Exp. 3 \\
\hline MinFluo & 0.984 & 0.987 & 0.77 \\
Commercial & 0.384 & 0.973 & 0.53 \\
\hline
\end{tabular}

(Fig 5b). Compared to the standard curve fitted on the $C_{t} \mathrm{~s}$ estimated by the commercial software (Fig 5a), our method MinFluo achieves better linearity. The same result is replicated across multiple experiments (table I).

MinFluo improves linearity likely due to the removal of the process noise represented by free adaptors, aptamers and leptin left in the solution, which can interfere with the PCR. By including a control, this process noise can be removed thus improving the sample quantification.

Moreover, the MinFluo method allows for implementation in real time and given its simplicity, it can be easily implemented on a portable PoC device.

\section{CONCLUSion}

In this paper we have presented the design methodology of a fully automated system for sensitive protein quantification in a PoC setting. We described design considerations and challenges associated to the implementation of the cartridge for sample preparation and of the machine for RT-PCR. Furthermore we presented preliminary results from the quantification methodology followed by a simple data processing algorithm for self-calibrated quantfication of the unknown sample. This system can be used as a PoC device for protein quantification, which has many applications ranging from personalised healthcare for example through close monitoring of hormonal changes, to disease diagnosis through detection of protein biomarkers such as antibodies and receptors.

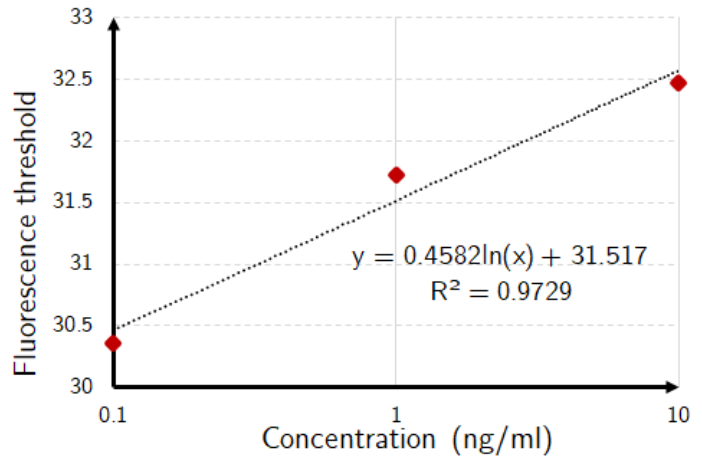

(a)

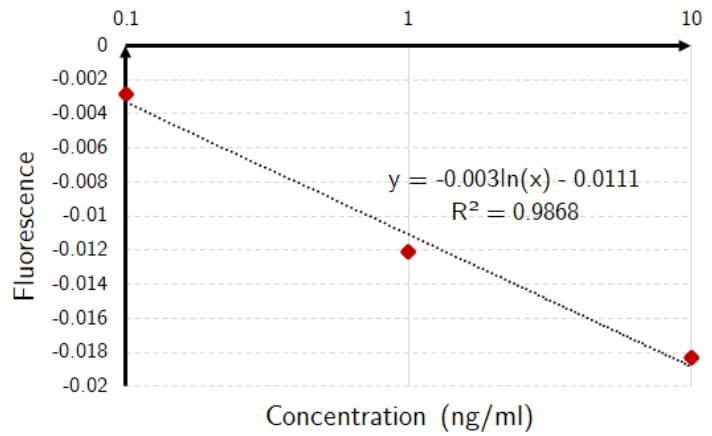

(b)

Fig. 5: Standard curves from leptin standards with the $\mathrm{Ct}$ method and without noise removal (a), and with the minimum fluorescence method plus noise removal (b).

\section{REFERENCES}

[1] A. Larsson, R. Greig-Pylypczuk, and A. Huisman, "The state of pointof-care testing: A european perspective," Upsala Journal of Medical Sciences, vol. 120, no. 1, pp. 1-10, 2015.

[2] A. E. Cass and Y. Zhang, "Nucleic acid aptamers: Ideal reagents for point-of-care diagnostics?" Faraday Discussions, vol. 149, pp. 49-61, 2011.

[3] K. Mullis, F. Faloona, S. Scharf, R. Saiki, G. Horn, and H. Erlich, "Specific enzymatic amplification of DNA in vitro: The polymerase chain reaction," Cold Spring Harbor Symposia on Quantitative Biology, vol. 51, no. 1, pp. 263-273, 1986. [Online]. Available: https://pubmed.ncbi.nlm.nih.gov/3472723/

[4] C. D. Ahrberg, B. R. Ilic, A. Manz, and P. Neužil, "Handheld real-time PCR device," Lab on a Chip, vol. 16, no. 3, pp. 586-592, 2016. [Online]. Available: www.rsc.org/loc

[5] C. Koo, M. Malapi-Wight, H. S. Kim, O. S. Cifci, V. L. VaughnDiaz, B. Ma, S. Kim, H. Abdel-Raziq, K. Ong, Y. K. Jo, D. C. Gross, W. B. Shim, and A. Han, "Development of a real-time microchip PCR system for portable plant disease diagnosis," PLoS ONE, vol. 8, no. 12, p. e82704, 12 2013. [Online]. Available: https://dx.plos.org/10.1371/journal.pone.0082704

[6] P. Neuzil, C. Zhang, J. Pipper, S. Oh, and L. Zhuo, "Ultra fast miniaturized real-time PCR: 40 cycles in less than six minutes," Nucleic Acids Research, vol. 34, no. 11, pp. e77-e77, 6 2006. [Online]. Available: http://www.appliedbiosystems.com/

[7] G. Mulberry, K. A. White, M. Vaidya, K. Sugaya, and B. N. Kim, "3D printing and milling a real-time PCR device for infectious disease diagnostics," PLOS ONE, vol. 12, no. 6, 2017. [Online]. Available: https://doi.org/10.1371/journal.pone.0179133

[8] C. Toumazou, S. B. Lowe, S. W. Green, P. S. Harding, G. H. W Sanders, N. J. Wooder, A. A. Werdich, M. Rene Clemens Twisk, R. J. Z. Heinz, J. Casey, H. V. Hare, R. Lintern, S. Weichert, S. James, K. Herbst, and L. Zanchet, "Method and Apparatus for Analysing a Biological Sample: Patent No: US 10,093,965 B2," 2018. 
[Online]. Available: https://patentimages.storage.googleapis.com/e6/c2/ 36/e1fc24e6588746/US10093965.pdf

[9] F. R. Cavallo, K. Mirza, S. de Mateo, K. Nikolic, J. Rodriguez-Manzano, and C. Toumazou, "Aptasensor for simultaneous quantification of leptin and short DNA-aptamers using qPCR," [Under Review], 2020.

[10] Y. Zhou and L. Rui, "Leptin signaling and leptin resistance," Frontiers of Medicine, vol. 7, no. 2, pp. 207-222, 2013. [Online]. Available: https://link.springer.com/content/pdf/10.1007/s11684-013-0263-5.pdf

[11] A. Dhiman, P. Kalra, V. Bansal, J. G. Bruno, and T. K. Sharma, "Aptamer-based point-of-care diagnostic platforms," pp. 535-553, 2017. [Online]. Available: http://dx.doi.org/10.1016/j.snb.2017.02.060

[12] J. Tellinghuisen and A. N. Spiess, "Comparing real-time quantitative polymerase chain reaction analysis methods for precision, linearity, and accuracy of estimating amplification efficiency," Analytical Biochemistry, vol. 449, no. 1, pp. 76-82, 2014. [Online]. Available: http://dx.doi.org/10.1016/j.ab.2013.12.020

[13] S. Zhao and R. D. Fernald, "Comprehensive algorithm for quantitative real-time polymerase chain reaction," Journal of Computational Biology, vol. 12, no. 8, pp. 1047-1064, 10 2005. [Online]. Available: www.liebertpub.com

[14] M. Guescini, D. Sisti, M. B. Rocchi, L. Stocchi, and V. Stocchi, "A new real-time PCR method to overcome significant quantitative inaccuracy due to slight amplification inhibition," BMC Bioinformatics, vol. 9, no. 1, pp. 1-12, 72008 [Online]. Available: https://link.springer.com/articles/10.1186/14712105-9-326https://link.springer.com/article/10.1186/1471-2105-9-326

[15] M. M. Gibani, C. Toumazou, M. Sohbati, R. Sahoo, M. Karvela, T.-K. Hon, S. De Mateo, A. Burdett, K. Y. F. Leung, J. Barnett, A. Orbeladze, S. Luan, S. Pournias, J. Sun, B. Flower, J. Bedzo-Nutakor, M. Amran, R. Quinlan, K. Skolimowska, C. Herrera, A. Rowan, A. Badhan, R. Klaber, G. Davies, D. Muir, P. Randell, D. Crook, G. P. Taylor, W. Barclay, N. Mughal, L. S. P. Moore, K. Jeffery, and G. S Cooke, "Assessing a novel, lab-free, point-of-care test for SARS-CoV-2 (CovidNudge): a diagnostic accuracy study," The Lancet Microbe, 9 2020. [Online]. Available: www.thelancet.com/microbeVol

[16] W. Lee, Y. M. Chae, S. Kim, S. H. Ho, and I. Choi, "Evaluation of a mobile phone-based diet game for weight control," Journal of Telemedicine and Telecare, vol. 16, no. 5, pp. 270-275, 2010. [Online]. Available: https://journals.sagepub.com/doi/pdf/10.1258/jtt.2010.090913 